\title{
Propagande dynastique et célébrations princières, mythes et images à la cour timouride
}

Dynastic Propaganda and Princes' Celebration

$$
\text { الدعاية للسلالة الحاكمة والاحتفال بأمرائها }
$$

\section{Anna Caiozzo}

\section{OpenEdition}

Journals

Édition électronique

URL : http://journals.openedition.org/beo/490

DOI : $10.4000 /$ beo. 490

ISBN : 978-2-35159-335-6

ISSN : 2077-4079

Éditeur

Presses de l'Institut français du Proche-Orient

Édition imprimée

Date de publication : 1 mai 2012

Pagination : 177-201

ISBN : 978-2-35159-193-2

ISSN : 0253-1623

Référence électronique

Anna Caiozzo, «Propagande dynastique et célébrations princières, mythes et images à la cour timouride », Bulletin d'études orientales [En ligne], Tome LX | mai 2012, mis en ligne le 31 mai 2012, consulté le 19 avril 2019. URL : http://journals.openedition.org/beo/490 ; DOI : 10.4000/beo.490 


\section{PROPAGANDE DYNASTIQUE ET CÉLÉBRATIONS PRINCIÈRES, MYTHES ET IMAGES À LA COUR TIMOURIDE}

Anna CAIOZZO

Université Paris 7 - Denis Diderot

Lorsque Tamerlan (1336-1405), autrement dit Tīmūr Lang (« le boiteux»), fils du chef d'une tribu turco-mongole de Transoxiane, conquiert le monde oriental à la fin du $\mathrm{XIV}^{\mathrm{e}}$ siècle, il fonde une dynastie qui devient très rapidement aussi glorieuse que celle des Gengiskhanides, à laquelle elle succède pendant près d'un siècle. Nouveaux venus sur la scène proche-orientale, régnant de l'Irak aux portes de la Chine et de l'Inde, les Timourides rêvent de conquérir ces deux puissances voisines comme ils ont soumis Bāyazīd et les derniers rois mongols, jalayrides de Bagdad et muzaffarides du Fars.

En un demi-siècle, les arts du livre connaissent en Iran et en Asie centrale un essor sans précédent : les princes timourides, avides de pouvoir mais aussi de culture, développent dans toutes les villes qu'ils gouvernent des ateliers princiers où la fine fleur des artisans, des calligraphes et des miniaturistes du monde musulman œuvrent pour eux. À la force brutale de la conquête se substituent des pouvoirs princiers collégiaux qui rivalisent en commandes architecturales et en collectes, commandes et achats d'œuvres d'art et de littérature.

Tīmūr Lang comprit quant à lui le rôle crucial des historiographes de cour dans la construction d'une légende personnelle bâtie dans la continuité d'ancêtres prestigieux voire mythiques. Cette démarche fut parachevée par ses héritiers qui à leur tour, soucieux à la fois de mettre en place un mythe familial héroïque et d'en récolter les fruits - dont la direction politique de l'empire ou de ses provinces - commanditèrent des ouvrages historiographiques dont certains illustrés, tel le célèbre Zafarnāme de 'Alī Yazdī dont seules trois copies nous sont malheureusement parvenues, l'une peinte en 1436, l'autre sous le règne de Sulțān Husayn Bayqarā’ vers 1480-1490, et une autre moins connue en $1486{ }^{1}$, et qui chacune s'accordent, par des styles différents et de façon complémentaire, à promouvoir l'image de Tīmūr comme chef politique, guerrier, bâtisseur, aristocrate et chef de clan. En

1. Zafarnāme, 1486, Turk ve Islam Ezerleri Muzesi, dans ROXBURGH 2005, fig. 172, p. 220. 
somme un roi des trois fonctions fidèle à l'idéologie des épopées indo-européennes dont le modèle dominant était alors le Shāhnāme du poète Firdaws $\overline{1}^{2}$.

Et, de fait, fiers de posséder un ancêtre fondateur d'un tel renom, les princes de la maison de Tīmūr renforcent à leur tour la légende familiale et hérö̈que à laquelle ils s'intègrent, tout en célébrant les valeurs de l'aristocratie iranienne portée par la geste des anciens rois de Perse, prestigieux modèles, dont les hauts faits sont représentés dans les copies enluminées du Shāhnāme. Cette épopée, fréquemment copiée à leur demande, gratifiée d'une introduction par le prince Bāysunghur, fut dotée de miniatures personnalisées et de frontispices répondant aux ambitions idéologiques des différents princes. En effet, si les fils et petits-fils de Tīmūr adoptent un idéal aristocratique commun, ils se distinguent chacun par des choix de gouvernement différents autour desquels ils bâtissent leur propre image du souverain idéal, image d'ailleurs fortement liée à leurs croyances aux astres.

De fait, de façon consciente et concertée, l'image, par le biais de la miniature mais aussi des fresques murales, allait devenir à l'époque timouride un instrument de promotion de la dynastie et des hommes.

\section{IMAGES DE TĪMŪR, ENTRE MYTHES ET LÉGENDE FAMILIALE}

1) L'acquisition d'une légitimité: de la promotion des supposés ancêtres fondateurs à la glorification de Tìmūr

Tīmūr était issu d'une tribu mongole à l'origine, mais turquisée par la langue et désormais islamisée ${ }^{3}$, les Bārlās ; admiratif de l'œuvre conquérante de Gengis Khan et de ses héritiers, il voulut légitimer l'installation au pouvoir de sa propre dynastie en la rattachant ouvertement à celle des Mongols. Cette réelle aspiration à retrouver une identité mongole - longtemps occultée par l'historiographie contemporaine souvent préoccupée par les problèmes de légitimité depuis René Grousset ${ }^{4}$ - permet de mieux comprendre certains des actes de gouvernement de Tīmūr. Il se présente ainsi comme le rénovateur de la dynastie gengiskhanide et comme son légitime successeur, ce qui lui permet de maintenir, dans un premier temps, la fiction d'un pouvoir mongol en Asie centrale ${ }^{5}$.

Le règne glorieux de Tamerlan permit à la nouvelle dynastie de se doter d'une légitimité en partie redevable à ses hauts faits. Le fondement du pouvoir selon le modèle persan préislamique repose amplement sur l'investiture du souverain pourvu de la grâce divine que le concept de farr ou khvarna retranscrit de façon tout à fait éclairante ${ }^{6}$. Le charisme personnel de Tamerlan (qut), les pouvoirs mystérieux dont le créditent ses

2. Une anthologie aurait été peinte pour Tamerlan regroupant le Banū Guschaspnāme, le Faramurznāme et le Garschaspnāme. Elle est dispersée et répartie entre autres entre le compendium Oriental 2780 du British Museum de Londres et le ms. de Dublin, Chester Beatty Library, Per 114. Voir les images sur le site http://shahnama.caret.cam. ac.uk; et ARBERRY 1959, vol. 1, p. 31-32 et TITLEY 1977, p. 39-40.

3. FORBES MANZ 1989a, p. 1-14.

4. GROUSSET 1965, p. 486-489.

5. SUBTELNY 2007, p. 18-24.

6. SUBTELNY 2007, p. 11-15. 
biographes évoquent des faits miraculeux relatifs à sa karāma, mais, surtout, éclairent littéralement le pouvoir d'une lumière divine et, de ce fait, le rendent incontestable ;image « rayonnante » mais relativement contrariante pour les pouvoirs religieux qui auraient préféré subordonner la salțana à la wilāya comme l'explique B. Forbes Manz ${ }^{7}$. Il s'ensuivit dans les milieux lettrés une véritable réflexion autour du pouvoir qui amena une partie des autorités religieuses à reconnaître la prééminence du chef «charismatique » dans le souci de conserver leur rôle à ses côtés et de continuer à bénéficier de son soutien tout en se méfiant d'un souverain qui leur disputait clairement leurs pouvoirs, leurs prérogatives et leurs compétences ${ }^{8}$.

La légitimité trouvait d'autre part l'un de ses fondements dans la gestion de l'héritage gengiskhanide et dans la volonté de Tamerlan ${ }^{9}$ d'affirmer sa fidélité à l'ancienne dynastie régnante par une série de traits sans équivoque allant de la recréation d'un empire à l'établissement de liens de sang avec les princesses gengiskhanides en passant par la temporaire institution d'un "khān fantoche » en début de règne ${ }^{10}$, en faisant faire la khutba au nom des descendants de Gengis Khan, et en se faisant nommer kürägän (beau-fils) lorsqu'il épouse, en 1370, une princesse mongole. Il donne d'ailleurs en mariage à presque tous ses fils et petits-fils des jeunes filles mongoles d'origine noble. L'adoption du yasa ou de la törä, sorte de constitution coutumière veillant à maintenir les lois turco-mongoles, est sans aucun doute le geste le plus éclairant qui distinguait clairement la famille timouride et les élites du reste de la population ${ }^{11}$. Mais l'un des éléments permettant de justifier cette volonté de se rattacher à l'héritage mongol est la place et le rôle affectés à l'origine au clan Bārlās : en effet, Qarashar Noyan fut affecté par Gengis Khan lui-même à la direction d'un contingent d'élite, le keshik, ce qui aurait promu les Bārlās à une position de premier plan qui se mesure encore sous Ghāzān Khān (1295-1304) ${ }^{12}$ et jusqu'à l'époque de Tamerlan par le service de l'ulu des descendants de Shaghatay ${ }^{13}$. Le keshik aurait été une pièce centrale des institutions mongoles, héréditaire et stable, qui permit l'émergence de l'État timouride porté par les émirs et la fidélité qu'ils devaient à leur chef ${ }^{14}$, et la légitimité du pouvoir militaire au sein duquel s'affirme la puissance de Tamerlan ${ }^{15}$.

7. FORBES MANZ 2007, « The ruler and the supernatural », p. 190-193.

8. PAUL 1990, p. 307-318.

9. C'est ce que suggère M. Bernardini en analysant les arguments de John Wood (BERNARDINI 2008, p. 52-53). M. Bernardini évoque aussi la tradition ghaznévide dont les souverains par souci de légitimité épousèrent des princesses iraniennes mais, comme il le dit lui-même, le modèle turc et « l'aturquitude » étaient à éviter de la part des Timourides.

10. FORBES MANZ 2007, p. 9-10 et SUBTELNY 2007, p. 23.

11. SUBTELNY 2007, p. 15-18.

12. MELVILLE 2006, p. 150-155.

13. SUBTELNY 2007, p. 20 et GRUPPER 1995, p. 84.

14. Sur le rôle des émirs, voir SHIRO 1992.

15. SUBTELNY (2007,p. 22) résume bien la situation : « As the descendant of the head of Chaghatay Khan's hereditary imperial guard corps, Temür was thus not a rootless parvenu but the scion of one of the great governing families of the Mongol Empire ». 
Cette volonté de s'inscrire dans la filiation mongole se retrouve également chez tous ses héritiers mais de façon parfois différente. Son fils Shāh Rukh (1377-1447) fit composer une généalogie, Mu'izz al-ansāb fì shajarat al-ansāb (La gloire des généalogies et l'arbre des origines familiales), en 1426 (traduite du turc au persan), continuation de la généalogie de celle proposée au XIII ${ }^{e}$ siècle par l'historien Rashīd al-Dīn dans son Histoire universelle ; cette dernière affirme clairement l'existence d'une ancêtre commune, la mystérieuse Alan Qoa ${ }^{16}$. Néanmoins, Shāh Rukh serait le seul - tout au moins officiellement - à s'être démarqué de l'héritage législatif mongol au profit d'une restauration sunnite en rétablissant pour tous la sharía et en se dotant du titre de pādishāh-i islām, en s'inspirant visiblement du modèle légué par le premier khān musulman Ghāzān ${ }^{17}$. Toutefois, le renforcement de l'orthodoxie sous Shāh Rukh semble à nuancer : pour partie imputable à une construction des historiographes de son temps, il reflète une réaction de reprise en main religieuse face à l'agitation de certains mouvement ésotériques qui menaçaient alors la stabilité de l'État, et on assista dans les faits à une application plus que modérée ${ }^{18}$.

Certains des petits-fils de Tamerlan gardèrent cette fidélité culturelle vis-à-vis des Mongols, puisqu'une autre généalogie fut commandée par Khalīl Sultān (m. 1411) ${ }^{19}$; préservée dans l'album Hazine 2152 (Istanbul, TSL, ms. H. 2152, fo 32b à 42a), elle présente les lignages timourides et celui de Gengis Khan comme issus d'un ancêtre commun Tüminay, lui-même descendant de l'ancêtre éponyme Alan Qoa, mère de Bodanchar et mythique fondatrice de la lignée ${ }^{20}$.

La filiation fut néanmoins « amendée » par Ulugh Beg (1394-1449), un autre de ses petitsfils, qui fit ajouter sur la tombe en jade noir de son grand-père une généalogie islamisée cette fois, où l'on apprend qu'Alan Qoa et 'Alī b. Abū Țālib faisaient conjointement partie des ancêtres du grand Tīmūr ${ }^{21}$. Dans l'Histoire secrète des Mongols, Alan Qoa explique qu'elle conçut ses trois fils par le biais d'une intense lumière jaune matérialisée sous l'aspect d'un homme venant à elle tel un esprit saint ${ }^{22}$; et l'inscription d'Ulugh Beg de préciser que cette lumière était l'essence même de l'un des fils de 'Alī ${ }^{23}$ !

Ainsi, l'élaboration d'une généalogique fictive permit à la fois la promotion de la famille timouride dans l'aristocratie mongole - historique ou mythique - et son intégration dans l'islam avec à première vue une forte connotation shi'ite liée aussi à la personnalité de Álī et à la diffusion d'une abondante littérature relatant ses hauts faits guerriers sur le

16. DOBROVITS 1994, p. 269-277.

17. AMITAI-PREISS 1996, p. 1-10; FORBES MANZ 1989, p. 28 et 2000, p. 33-47.

18. SUBTELNY 2007, p. 26.

19. WOODS 1987.

20. Mongol-Timurid Genealogy as Given in Istanbul, TSM H.2152, « Genealogical Charts », dans THACKSTON 1989, p. XVI; Woods 1990.

21. AIGLE 2000 c, en particulier $\S 4$ et 5 et « The Transformation of an Origin Myth from Shamanism to Islam », dans Aigle (à paraître).

22. Histoire secrète des Mongols, Chronique mongole du XIII' siècle, p. 44.

23. LENTZ et LOWRY 1989, p. 28. 
mode épique, ce qui assurait la promotion de Tamerlan comme combattant «sanctifié ", à l'image d'une autre grande figure, 'Alī, que l'on comparait inconsciemment ou non au grand héros Rustam ${ }^{24}$.

Les Timourides ainsi officiellement rattachés aux Mongols furent dès lors investis d'un devoir de mémoire vis-à-vis de leurs prédécesseurs et, afin de célébrer la geste héroïque et conquérante de cette dynastie, commandèrent des exemplaires illustrés de manuscrits historiques telles les œuvres de Hāfiz-i Abrū, Majma' al-tawārìkh (Le Rassemblement des histoires, Istanbul, TSL, Hazine 1653) ou Kuliyyat-i Tarikh (La Somme de l'Histoire, Istanbul, TSL, ms. Bagdad $282{ }^{25}$ ), mais aussi l'Histoire des Mongols de Rashīd al-dīn, dont une copie illustrée est aujourd'hui conservée à Paris à la BnF. (ms. sup. persan 1113, Planche $1{ }^{26}$ ). Le manuscrit, peint à Hérat vers 1430-1434, offre une galerie de portraits de Gengis Khan à Ghazān, de leurs exploits militaires, tableaux édifiants offerts aux regards des petits-fils de Tīmūr.

Tīmūr Lang nous est connu par ses nombreux biographes et par les restes de ses ossements trouvés il y a soixante ans par Guerassimov dans son sarcophage du Gūr-i Amīr (Tombeau de l'émir) ${ }^{27}$. Ce sont ses biographes, voire ses historiographes, qui en dressent cependant le portrait le plus fidèle. Certes, d'après Ibn 'Arabshāh, biographe autant que détracteur, il était illettré ; pourtant, Ibn Khaldūn, qui le rencontra en 1402 avant la conquête de l'Anatolie, le décrivit dans ses mémoires comme un homme vif, intelligent, ayant parfaitement intégré le sens de l'histoire ${ }^{28}$. Ibn 'Arabshāh lui-même souligne combien il aimait qu'on lui lise l'Histoire des prophètes, des rois du passé, y compris ceux de l'ancienne Perse. Cet intérêt pour le passé, le sien et celui des autres, aida non seulement à la confection d'une lignée aux ancêtres mythiques, mais aussi à l'écriture d'une histoire personnelle puis familiale, partagée entre rêve et réalité. Car Tīmūr se faisait accompagner par des secrétaires iraniens ou ouighours qui notaient ses moindres faits et gestes, dans un souci historiographique évident. Patiemment, les Timourides bâtissaient leur légende ${ }^{29}$. Toutes les périodes de la vie de Tīmūr ont ainsi été répertoriées par ses biographes ou historiographes officiels, mais aussi par des chroniques étrangères (celle de Ruiz de Clavijo, entre autres). Certaines opérations militaires entre 1386 et 1393 sont retracées par des témoins éminents, souverains étrangers tel Zayn al-Dīn Qazwīnī, décrivant le règne de son père Ḥamd Allāh (Tārîkh-i Guzīde) ou Burhān al-Dīn Aḥmad, souverain de Sivas entre 1380 et

24. AIGLE 2000c., § 23-24 et MELVILLE 2006.

25. INAL 1992, p. 103-115.

26. RICHARD 1997a, p. 307-320 et RICHARD 1997b, notice 40, p. 76.

27. LENTZ et LOWRY 1989, p. 28, fig. 3.

28. Fischel 1952, p. 47 : «Le souverain Timur est l'un des plus grands et le plus puissant des rois, certains lui attribuent le savoir et la connaissance, d'autres le disent hérétique à cause de sa préférence des membres de la famille d'Alī, d'autres encore lui attribuent l'emploi de la magie et de la sorcellerie mais de tout cela il n'y a rien ; il est simplement très intelligent et perspicace, et il participe au débat et argumente, sur ce qu'il sait et sur ce qu'il ne sait pas. »

29. WOODS 1987. 
1398, qui raconta les incursions timourides dans la région, mais aussi par les chroniqueurs mamelouks ${ }^{30}$...

L'une des plus célèbre biographies connues est le Zafarnāme ou Histoire glorieuse de Tìmūr. On en compte plusieurs versions : celle de Șafī al-Dīn Kuttalanī Samarqandī en turc, mais surtout celle de 'Alī Shāmī, écrite entre 1401 et 1404, ouvrage de commande à portée apologétique, insistant sur la piété et sur l'intégration du conquérant et de sa famille à l'islam, comme l'atteste d'ailleurs l'ensemble du patronage architectural (nombreux mausolées en l'honneur de chefs soufis, dont Ahmad Yasavī, mort en $1166^{31}$ ). Tīmūr y est décrit comme visitant les pieux personnages, accompagné de sayyīds, et toujours pourvu dans ses déplacements de scribes ouighours et de secrétaires persans ${ }^{32}$.

Cette biographie subit des amendements à la fois de son vivant et, par la suite, entre 1419 et 1426. Hââiz-i Abrū, qui avait fréquenté la cour du vivant de Tīmūr, avait inclus la biographie de Shamī à son compendium ou Majma' al-tawārīkh demandé par Shāh Rukh en $1417^{33}$. Par la suite, il travailla pour Ibrāhīm Sulțān (1394-1435) et Bāysunghur (1397-1434), et en 1426-27, en prenant comme base l'Histoire des Mongols de Rashīd al-Dīn : il insista bien sur les origines mongoles de Bodanchar tout en intégrant dans la section finale de son œuvre, dédicacée à Baysunghur, le Zubdat al-tawārīkh ou Le Meilleur des histoires, la famille timouride ${ }^{34}$.

Sharaf al-Dīn 'Alī Yazdī laissa l'œuvre la plus connue de cette époque ${ }^{35}$, puisqu'elle fut composée et achevée entre 1419 et 1424 et que son écriture fut largement contrôlée par le commanditaire Ibrāhīm Sulțān ${ }^{36}$. Réalisée à partir de matériaux variés amplement puisés chez Shamī, mais aussi de faits et gestes rassemblés dans ce but, écrite à la demande, déconnectée de la réalité, l'œuvre n'arrive pas à masquer le paradigme dominant: une volonté de respecter la tradition et le pouvoir mongol et les faits qui s'en démarquent par une évidente appropriation ${ }^{37}$. Par ailleurs, Ibrāhīm Sultān y fit intégrer en 1424 des poèmes et des épisodes de la vie de son père, Shāh Rukh, privilégiant une lignée aux dépens de celles de 'Umar Shaykh et de Miranshāh dans la tradition inaugurée en 1410 par Tāj al-Dīn al-Salmānī qui composa le Shams al-Husn (Le soleil de la beauté), en son honneur. Le Zafarnāme présente ainsi, dans sa version illustrée de 1436, des scènes de la vie de Tīmūr et cinq scènes de celle de Shāh Rukh.

Pourtant il semblerait que la compilation de Sharaf al-Dīn Yazdī soit largement redevable à l'œuvre d'un scribe autrefois au service des Muzaffarides de Yazd, Ghiyāth al-

30. WOODS 1987, p. 84.

31. LENTZ et LOWRY 1989, p. 40-41.

32. LENTZ et LOWRY 1989, p. 85-86.

33. LENTZ et Lowry 1989, p. 96-99.

34. FORBES MANZ 2007, p. 41-42.

35. FORBES MANZ, 2007, p. 99-105. Sharafuddīn 'Al̄̄ Yazdī, Zafarnama, p. 63-100, Niz̄ām al-Dīn 'Alī Shāmī, Zafar-nāme. Ce Zafarnāme possède la particularité d'intégrer la généalogie mythique des Timourides telle que le Mu izz al-ansab, celle d'Ibrāhīm Sulțān ou celle d'Ulugh Beg.

36. WOODS 1987, p. 100-102.

37. WOODS 1987, p. 105. 
Dīn 'Alī Yazdī qui écrivit durant le règne de Tamerlan, entre 1401 et 1403, la campagne en Inde dans ses Ghazavāt-e Hindūstān, suivies du Sa'ādatnāme, ou Livre de la fortune. Cette œuvre relatant des faits de ghazavāt ou expéditions liées à la guerre sainte, rattachait Tamerlan à la tradition de la ghazwa et des guerres menées naguère par le Prophète, et la rehaussait ainsi d'une aura de combattant de la foi triomphant des Indiens mécréants et idolâtres ${ }^{38}$.

Les spécialistes du monde timouride - de John Woods à Denise Aigle en passant par Maria Subtelny ou Beatrice Forbes Manz et bien d'autres encore - s'accordent à penser que les chroniqueurs ont fait de Tamerlan une icône, dynaste idéal, guerrier parfait, de corps comme d'esprit, minimisant tant ses défauts physiques que moraux par des procédés rhétoriques et analogiques avec les héros d'épopée, et par l'affirmation que sa geste remarquable était guidée par les cieux ${ }^{39}$.

\section{2) Le Zafarnāme ou la mise en scène d'un héros}

Le Zafarnāme offre le témoignage visuel de cette doxa reproduite dans plus de trente copies illustrées, bien que seuls deux manuscrits principaux soient aujourd'hui connus et étudiés, celui de 1436, dispersé entre plusieurs collections, et celui de la Garett Collection, commissionné à la fin du XV $\mathrm{XV}^{\mathrm{e}}$ siècle par Sulțān Ḥusayn, le dernier Timouride d'Hérat ${ }^{40}$.

Les miniatures du Zafarnāme de 1436, tout comme celles de 1480, nous livrent un portrait idéal de Tīmūr, guerrier et conquérant, diplomate et aristocrate ${ }^{41}$. Ainsi, le 10 avril 1370, il s'intronise lui-même maître de Balkh et cumule la Transoxiane, où il a installé des khāns fantoches de la dynastie gengiskhanide, à l'Afghanistan ${ }^{42}$. Cette scène inaugure le cycle illustratif du Zafarnāme de 1436 et consacre l'image du souverain accueillant ses courtisans dans un jardin, selon un protocole que l'on devine très rigide, comme l'explique Clavijo à longueur de pages ${ }^{43}$, et la position des membres est révélatrice du rang occupée : les jambes croisées pour les princes royaux, un genou replié pour les autres, à genoux pour les vassaux ${ }^{44}$. Il est à noter que la seule représentation de Tīmūr à genoux en position d'infériorité vis-à-vis des ancêtres se trouve dans la généalogie conservée à Topkapi (ms. $\mathrm{H} 2152)^{45}$.

38. Ghiyāsoddīn 'Alī di Yazd, Le Gesta di Tamerlano, p. xxv-XxxII, d'après la version de Ghiyāth al-Dīn 'Alī Yazdī, Sa'ādatnāma yā Rūz-nāme-ye Ġazavāt-e Hendūstān.

39. POLYAKOVA 1988, p. 31-44.

40. Sharaf al-Dīn 'Alī Yazdī, Zafarnnama, Hérat, copié en 1467, peint en 1480, Baltimore, John Hopkins University, Milton S. Eisenhower Library, John Work Garett Collection (voir SIMS 1973).

41. SIMS 1990-91, 36 peintures.

42. SIMS 1990-91, p. 185. Folios 128v-129 : Washington D.C., The Arthur Sackler Gallery, S86.0133.001-S86.0133.002. Zafarnāme, p. 202 : «Timur monta sur le trône, mit la couronne d'or sur sa tête, se ceignit lui-même de la ceinture impériale en présence des princes du sang royal et des émirs qui se mirent à genoux et l'ayant félicité lui firent de magnifiques présents et répandirent à pleines mains sur sa tête des quantités d'or et de pierreries selon la coutume et lui donnèrent le titre de Sahib Qiran et dès lors tout le pays fut assujetti à ses lois. »

43. CLAVIJO, La Route de Samarkand au temps de Tamerlan, 1403-1406, p. 210.

44. NECIPOǦLU 2000, p. 22-61.

45. NECIPOǦLU 2000, p. 25. 
Pourtant, sur trente-six miniatures, la moitié est réservée à la guerre, proportion bien inférieure au texte du Zafarnāme qui, lui, consacre l'essentiel de son propos aux campagnes. Le choix de l'image estévident : outre un guerrier, Tīmūr est aussi un politique, un aristocrate et un chef de clan, comme il s'évertue à le démontrer aux ambassadeurs castillans.

Les illustrations à caractère militaire concernent les triomphes majeurs du règne sur les derniers dynastes mongols, les émirs de Syrie et le sultan ottoman. Les victoires sur Toqtamish, chef de la Horde du Qipchaq en Russie méridionale, en $1391^{46}$, celle sur Shāh Manșūr Muẓaffar en Azerbaïdjan en mars 1393, la campagne menée contre le sultan indien Mạ̣mūd Khān Tughluq en décembre $1398{ }^{47}$ consacrent de grands triomphes tant politiques que matériels. Puis sont présentées de façon plus détaillée les conquêtes occidentales : campagnes contre les Mamelouks en Syrie et en Mésopotamie, dont l'attaque et le siège d'Alep en novembre $1400^{48}$; la seconde chute de Bagdad en août 1401, au cours de laquelle le Mongol Sulțān Aḥmad Jalayr fuit en Égypte ${ }^{49}$. La campagne contre les Ottomans, qui valut à Tīmūr la présence d'observateurs castillans et dont les répercussions furent grandes en Occident, est représentée dans quatre miniatures et débute par la revue des troupes à Ankara au milieu de l'été $1402{ }^{50}$, la mise en présence des deux armées et de leurs chefs respectifs, Beyāzīt et Tīmūr, en juillet $1402{ }^{51}$. Deux épisodes particuliers sont signalés pour leurs prouesses : l'attaque du château de Gortīn réputé imprenable ${ }^{52}$, et le siège des Abkhazes réfugiés dans des grottes en août $1403^{53}$. L'ultime scène de guerre est symbolisée par le départ de Shāh Rukh pour la Chine en février 1405, précédé par une troupe de hérauts, départ dont le caractère guerrier est totalement gommé ${ }^{54}$.

Toutes les scènes de guerre sont construites de façon identique : Tīmūr à cheval, isolé de la scène ou des autres personnages par un élément de relief, à l'écart de ses troupes, supervise les combats qui se limitent aux sièges de villes, ou à des batailles rangées entre deux armées identiques. En dehors des chefs concurrents, Toqtamish ou Beyāzìt, aucun soldat n'est repérable. Tous forment la masse des inconnus anonymés par un heaume conique. Le nombre de miniatures dédiées à la guerre s'explique certes par la renommée militaire de Tamerlan, mais aussi, par la place de cette activité dans l'État mongol puis

46. SIMS 1990-1991, fig. 8/9, p. 187. Folios 207v-208r: Genève, Hashem Khosrovani Collection, T/128. Sur l'affrontement entre Tīmūr et Toqtamish, voir FORBES MANZ 2000, p. 129-149 et § 21-32.

47. SIMS 1990-1991, fig. 14, p. 188. F. 290v : Gênes, The Bruschettini Collection.

48. SIMS 1990-1991, fig. 17/18, p. 189. F. 331v-332r. 17 : collection privée ; 18 : collection privée.

49. SIMS 1990-1991, fig. 21/22 p. 190. F. 345V-346r. 21 : New York City, The Metropolitan Museum of Art ; 22 : New York City, The Metropolitan Museum of Art, 67.266.1.

50. SIMS 1990-1991, fig. 23/24, p. 191. F. 355v-356r. 23 : collection privée ; 24 : The Keir Collection III.79.

51. Sims 1990-1991, fig. 25/26, p. 191. F. 359v-360r. 25 : localisation inconnue ; 26 : Jérusalem, The L.A. Mayer Memorial Institute for Islamic Art, 23-68.

52. SIMS 1990-1991, fig. 29/30, p. 192. F. 380v-381r. 29 : New York City, The Metropolitan Museum of Art, 55.121.16 ; 30 : collection privée.

53. SIMS 1990-1991, fig. 31, p. 193. F. 384r : collection privée.

54. Sims 1990-1991, fig. 36/37, p. 194. F. 413v-414r: Téhéran, The Reza Abbāsī Museum, Mehdi Mahboubian Collection. 
timouride, comme l'explique fort justement René Grousset : faute d'hommes pour pacifier durablement les régions, les victoires de Tīmūr étaient temporaires, et ses campagnes étaient pour ainsi dire permanentes. Ainsi, le long conflit contre Toqtamish dans lequel le récit du Zafarnāme se perd. Et, de fait, faute de moyens pour soumettre et administrer les territoires, Tīmūr ne laissa à sa mort que l'Iran et l'Asie centrale à ses descendants ${ }^{55}$.

Pourtant, contrairement aux illustrations de L'Histoire des Mongols, également commanditée par l'un de ses petits-fils (Paris, B.n.F., ms. sup. persan $1113^{56}$ ), les peintres du Zafarnāme ignorent les scènes de massacres ${ }^{57}$ et l'image reste dans la célébration la plus convenue. Les biographes contemporains (Zafarnāme, Clavijo ${ }^{58}$ ) ou postérieurs, tel Khwandamir, n'hésitent cependant pas à évoquer la terreur que le nom même de Tīmūr inspirait aux populations de l'empire ou les massacres de populations qu'il perpétra, comme celui de Bagdad en $1401^{59}$. Comme l'a montré Jean Aubin ${ }^{60}$, ou plus récemment Beatrice Forbes Manz ${ }^{61}$, Tīmūr massacrait - dans la plus pure tradition mongole - les habitants des villes et régions quilui résistaient: Balkh, Bagdad, Damas, le Séistan, etc. Des scènes d'horreur sont décrites par le Zafarnāme mais aussi par Ibn 'Arabshāh, confirmées par le témoignage de Clavijo vingt ans plus tard: fortifications démantelées, villes détruites, montagnes de corps humains ou de têtes ayant servi à édifier des tours d'hommes et d'argile ${ }^{62}$ ! La terreur utilisée comme mode de gouvernement est évidemment ignorée par l'image.

On peut de façon générale noter l'absence totale de sang, de morts ou de blessés au combat, et donc, en somme, la promotion d'une sorte de guerre propre et idéale offerte au regard, identique à bien des scènes de batailles du Shāhnāme, Le Livre des rois de l'ancienne Perse, dont un grand nombre d'exemplaires furent commandés par ses petits-fils ${ }^{63}$. Cette glorification d'une guerre complètement dématérialisée est associée à l'une des caractéristiques majeures du portrait du conquérant dont les traits sont figés dans toutes les miniatures connues, y compris dans les scènes de 1403, alors que Clavijo parle ici d'un vieillard ${ }^{64}$. Là encore, étrangement, l'Histoire des Mongols montre de façon plus réaliste l'évolution physique de Gengis Khan en compagnie de sa famille (jeune, dans la force

55. GROUSSET 1965, p. 497-498.

56. Un manuscrit de l'Histoire des Mongols de Rashīd al-Dīn, enluminé à Hérat vers 1440 sous Bāysunghur, petit- fils de Tīmūr, nous montre sans pudeur et restriction ces scènes d'horreur perpétrées par les grands ancêtres dont se réclament les Timourides (Paris, B.n.F., sup. persan 1113, f. 242v, 43v, 98v, 71v).

57. 'Al̄̄ Yazdī, Zafarnāme, livre 5, volume 3, p. 151 ; livre 4, volume 3, ceux des païens et idolâtres indiens, ceux des révoltés, ceux des shi'ites, etc.

58. CLAVIJO, La Route de Samarkand, p. 181.

59. Khwandamir, Habib al-Siyar, dans THACKSTON 1989, p 105. Khwandamir, qui écrivit en 1523, était le petit-fils de Mirkhwand et il utilisa, outre ses travaux, ceux de 'Abd al-Razzāq Samarqandī et surtout les deux Zafarnāme d'Alī Yazdī et Shāmī.

60. AUBIN 1963, p. 83-122.

61. FORBES MANZ 2002, p. 1-25.

62. Zafarnāme, I, p. 377. Ibn 'Arabshāh, Tamerlane, p. 25-27 ; CLAVIJO, La Route de Samarkand, p. 176.

63. Voir infra.

64. CLAVIJO, La Route de Samarkand, p. 210 ; Tīmūr fait approcher les ambassadeurs pour les voir de près car sa vue n'est pas bonne : il était d'un grand âge et ses paupière lui tombaient sur les yeux. 
de l'âge et vieillissant, les cheveux et la barbe blanche, Paris, BnF., ms. sup. persan 1113, fol. $85 \mathrm{v}, 92,99 \mathrm{v}, 51 \mathrm{v}, 44 \mathrm{v}$ ).

La miniature célèbre par ailleurs le dynaste politique, chef de clan redouté et incontesté, qui règne, d'après les auteurs, en solitaire. En fait, les attributs visibles du pouvoir sont la couronne, l'épée, mais aussi le parasol ou le trône sur lequel il siège parfois ${ }^{65}$.

La miniature ne reproduit cependant pas une des dimensions du pouvoir: le cosmocrator. En effet, si divers vocables sont utilisés à son endroit (le plus courant, Amìr Ghazī ou " combattant de la foi », Sāhib Qiran, " Maître des sept planètes ») accompagnés de diverses épithètes dont « Soleil des cieux » ou « Pādishāh de l'islam » ${ }^{66}$, Tīmūr est aussi présenté en 1404 lors des cérémonies de mariage à Gan-i Gul comme un roi cosmique, car sa tente, décorée d'or et de joyaux, se dresse au dessous de la lune et du soleil et «dresse sa tête au milieu des Pléiades ». L'enceinte où il réside ressemble au paradis, et le toit de la tente figure la voûte céleste ${ }^{67}$. Le symbolisme astral est également présent sur son palais de l'Aq-Saray de Kish :

«Le fronton de ce portail porte la représentation d'un lion placé dans un soleil, au centre, et à chaque extrémité une figure identique ${ }^{68}$.

Outre la symbolique du ciel auquel il est souvent associé comme témoignage de l'attachement à la culture turco-mongole, les auteurs évoquent aussi « les quatre piliers » de son règne, à savoir ses quatre fils Jahāngīr Sulțān, 'Umar Shaykh Sulțān, Miranshāh Kuragan, Shāh Rukh Bahādur Ghazī ${ }^{69}$. Mais d'autres éléments font de Tīmūr plus qu'un roi cosmique sur le mode sassanide, une sorte de shaman mongol. En effet, le symbolisme de l'âme est associé dans le monde turco-mongol à l'aigle, oiseau psychopompe, forme que revêt parfois le shaman; ainsi dans une tente, il siège sous :

«L'ouvrage le plus riche du pavillon était son plafond qui portant dans ses quatre coins des représentations d'aigles aux ailes déployées ${ }^{70}$.»

et dans une autre :

«Au point le plus haut de la tente se trouvait un grand aigle en argent doré aux ailes déployées; sous lui, à une brasse et demie, trois faucons en argent doré étaient suspendus à des intervalles réguliers. Ils tendaient leurs ailes comme s'ils voulaient fuir l'aigle ; leurs têtes étaient tournées vers celui-ci, dont la posture indiquait qu'il voulait s'élancer vers l'un d'eux. La forme et l'attitude de ces rapaces étaient si bien rendues qu'ils ressemblaient à la perfection à leurs modèles vivants ${ }^{71}$. "

65. FORBES MANZ 1988, p. 107-118.

66. Khwandamir, Habib al-Siyar, dans THACKSTON 1989, p. 105.

67. Zafarnāme, p. 91-92.

68. CLAVIJO, La Route de Samarkand, p. 199.

69. Mīr Dawlathshāh Samarqandī, Tadhkitar, dans THACKSTON 1989, p. 17.

70. CLAVIJO, La Route de Samarkand, p. 220.

71. Clavijo, La Route de Samarkand, p. 222. 
Par ailleurs, il est rapporté par Jean de Sultāniyye et d'autres auteurs que Tīmūr entendait des voix, que les anges lui parlaient et qu'il accédait aux cieux par une échelle! Loin de l'imitation de Muhammad, il faudrait sans doute y voir un rite plus proprement shamanique.

La glorification visuelle de Tīmūr, patente dans les scènes de majesté, compense cependant cette lacune, car elle permet la mise en exergue du personnage. Ces dernières, doublées d'un caractère politique, militaire ou diplomatique, comme l'investiture de Balkh ${ }^{72}$ ou la célébration du triomphe de Delhi en décembre $13988^{73}$, montrent le souverain isolé sur son tapis, ou sur un trône protégé par un parasol ou un auvent ${ }^{74}$ (à Ankara), voire à proximité de sa tente (Zafarnāme de 1480), assis jambes croisées ou à demi croisées, vêtu d'une robe verte et d'une chemise rouge, d'un chapeau mongol parfois surmonté d'une couronne, ou tenant le dastar ${ }^{75}$. Les scènes de majesté, diversifiées dans le détail par la remise de présents particuliers (la girafe de l'ambassade d'Égypte) ${ }^{76}$, la réception de vœux par l'un des émirs ${ }^{77}$, montrent Tīmūr doublement isolé par un cours d'eau, telle une frontière tangible, et un tapis ou un trône. Cet espace, quasi sacralisé, encerclé de gardes armés, n'est foulé que par ces derniers, dans la copie de 1480, voire par une épouse et un émir dans celle de 1436 ; si Tīmūr tend parfois la main droite en signe d'accueil ${ }^{78}$, les invités demeurent à bonne distance.

Quelques scènes du même registre se doublent du souci de le célébrer comme bâtisseur afin de témoigner de la politique architecturale et somptuaire du conquérant. Tīmūr détruisit beaucoup et, s'il construisit, ce fut surtout à sa propre gloire ou à celle de sa famille (somptueux palais, jardins, mosquées, tombeaux). En 1413, l'auteur anonyme d'une biographie synoptique des Timourides débute son récit par la prise de pouvoir de Tīmūr sur Amīr Husayn en disant : « il fit de Samarkand sa capitale et construisit un rempart, un palais et une citadelle ${ }^{79}$.»

Le Zafarnāme de 1436 évoque, outre la reconstruction de la cité de Baylaqān à l'automne $1403^{80}$, celle de son palais blanc, Aq-Saray, en $1379^{81}$ à Kish, sa ville natale, qu'il

72. Mīr Dawlathshah Samarqandī, Tadhkitar, dans THACKSTON 1989, p. 16v.

73. SIMS 1990-1991, fig. 15, p. 189, f. 293v, Cambridge, Harvard University, Fogg Art Museum, 1960.198.

74. BLAIR 1996, p. 562.

75. SOUDAVAR 2003 et BLAIR 1996, p. 552-554.

76. SIMS 1990-1991, fig. 34/35, p. 194, folios 399v-400r, The Keir Collection, PP5 (34) et The Worcester Art Museum, 1935-26 (35).

77. SIMS 1990-1991, fig. 13, p. 188, folio 274r, The Keir Collection, III.80. Les personnages politiques protégés de Tīmūr, tel Nūr al-Dīn Amīr Shaykh, régent du Fars, lui offrent souvent des présents en signe de soumission et de vassalité.

78. Balkh en 1370, revue des troupes à Ankara, accueil de Nūr a-Dīn Amīr Shaykh, fêtes de 1405, etc.

79. Anonymous, « Synoptic Account of the House of Timur », dans THACKSTON 1989, p. 237.

80. SIMS 1990-1991, fig. 32/33, p. 193. F. 386v-387r : collections privées. Zafarnāme, vol. 4, L. 6, p. 118.

81. Zafarnāme, vol. 1, p. 306-308: «La ville de Kish nommée Qubbāt al-ilm, la ville de la science et Shāh al-Sabz, la ville verte. Il la déclara second siège de son royaume et fit bâtir un nouveau palais qu'il fit nommer Aq-Sarayi (palais blanc) à cause de la blancheur prodigieuse de ses murs qui furent enfin extraordinairement exhaussés. Les fondements de ces édifices furent jetés durant l'heure convenable pour l'horoscope de la plus heureuse des cités et le palais fut bâti d'une si belle et si rare architecture qu'il ne s'en était jamais vu de semblable. Timur partagea ensuite 
dota d'une forteresse en $1381^{82}$. Par ailleurs, le Zafarnāme de 1480 témoigne du chantier « pharaonique » mis en œuvre pour la construction du Bibi Khanūm, en 1399, la grande mosquée de Samarkand construite en l'honneur de son épouse ${ }^{83}$.

\section{LE ZִAFARNĀME, DE LA GESTE DE TĪMŪR À LA PROMOTION D’UNE LIGNÉE}

Les miniatures du Zafarnāme de 1436, certes dédiées en premier lieu au conquérant, reflètent deux autres tendances majeures : les liens familiaux et le style de vie aristocratique d'un clan autrefois nomade.

Les biographes de Tīmūr insistent tous sur son intérêt pour sa progéniture et ses femmes. D’ailleurs, séparer dans la miniature le registre public du privé paraît extrêmement difficile; on note que certains grands événements familiaux, décrits de façon prolixe par le Zafarnāme ou par Clavijo, sont représentés dans une volonté délibérée de singulariser certains membres de la famille rattachés directement aux lignages mongols par mariage ou revendiquant la légitimité du pouvoir dès le décès du grand émir, à savoir Shāk Rukh.

Tīmūr montra tout au long de sa vie un intérêt particulier pour les siens. Les enfants nouveau-nés, fils et petits-fils, sont immédiatement enlevés à leur mère pour être confiés à des mères adoptives et à des précepteurs qu'il choisit lui-même. Il surveille leur éducation, affecte ses fils aux différentes parties de l'empire qu'il gouverne cependant en maître, et n'hésite pas à les punir durement en cas de désobéissance. Si Tīmūr eut un règne solitaire à ses débuts, il y associa très vite ses enfants comme chefs d'armée et gouverneurs de provinces; 'Umar Shaykh fut le premier à périr en 1392, au cours d'une campagne, en rejoignant son père dans l'ouest. La perte d'un proche ne laissait pas Tīmūr indifférent, comme en témoigne la déchirante scène de funérailles de Jahāngīr, son premier né, où l'on peut voir pleurer le conquérant ${ }^{84}$. Et la disparition de son petit-fils Muhammad Sulțān ibn Jahāngīr, au printemps 1403, l'affecta à nouveau profondément ${ }^{85}$.

la ville à ses émirs et aux troupes de sa maison. ». SIMs 1990-1991, fig. 5, p. 186. Copie de 1436, f. 153, localisation inconnue.

82. Anonymous, Synoptic Account, dans THACKSTON 1989, p. 238.

83. ARNOLD1930, pl.IXetX.Anonymous, Synoptic Account, dansTHACKSTON1989, p.239et'AlīYazdī,Zafarnāme, vol. 3, p. $178-180$.

84. Zafarnāme, p. 269-272.

85. Zafarnāme, p. 70. Il jeta la couronne, et abandonna le trône, il déchira ses vêtements et se jeta par terre, avec des transports et des lamentations étranges : «Les princes et les seigneurs de la cour se revêtirent de sacs de crin et d'habits noirs et bleus avec des feutres sur le cols, les dames en firent de même, et les uns et les autres jetèrent de la terre sur leur tête et remplirent leur sein de pierres ; ils ne voulurent se reposer que sur de la paille et de la cendre au lieu du matelas et l'on n'entendit plus à cette cour que des gémissements et des plaintes, principalement de la princesse Caniké épouse du défunt, si désolée, qu'elle jetait incessamment des cris et des lamentations, tombant à tout moment évanouie, et perdant le jugement ». Sims 1990-1991, fig. 27/28, p. 192. F. 373v-374r : Gènes, The Bruschettini Collection. 
La naissance de ses enfants fut source de réjouissances comme l'explique le Zafarnāme ${ }^{86}$, telle celle de Shāh Rukh en août 1377, présentée à dessein par le manuscrit de 1436 pour établir visuellement la filiation " légitime ${ }^{87}$ ».

Tīmūr célébra à grand frais le mariage de ses petits-fils aux fêtes de Can-i ghul en $140{ }^{88}$. Elles ne sont pas représentées mais seulement évoquées par l'arrivée de l'ambassade d'Égypte. En revanche, on le voit recevant des présents lors du mariage de Jahāngīr en juin 1374, lequel épousait alors Sevin Beg Khanzada, petite-fille de Janī Beg Khān, un prince de la maison de Jochi, fils de Čingis Khān ${ }^{89}$.

Les hauts faits de ses héritiers sont également représentés, telle l'entrée de Shāh Rukh, âgé de 17 ans, à Samarkand en 1394, alors qu'il venait de terrasser Shāh Manșūr Muzaffar ${ }^{90}$, puis le départ de l'armée timouride guidée par le même Shāh Rukh devenu chef de famille après la mort de Tīmūr.

Les épouses surnommées «les Belqīs du temps » sont, dans les sources, présentes à ses côtés ${ }^{91}$, mais si Tīmūr Lang eut dix-huit épouses recensées par Khwandamir et vingtet-une concubines, une seule fut représentée officiellement dans le manuscrit de 1436: Saray Mulk Hanim, en compagnie de son fils Shāh Rukh et de Khalīl Sulțān s'avançant sur la route de Marand en Azerbaïdjan en 1387. Cette épouse occupa un rang certain à ses côtés, et c'est en son honneur que la grande mosquée de Samarkand fut érigée comme le montrent les miniatures du Zafarnāme peint en 1480. Clavijo donna une description fameuse de cette première épouse qui, à l'image de son mari, offrait elle aussi des réceptions aux ambassadeurs ${ }^{92}$. Toutefois, d'autres épouses sont évoquées par les biographes: Touma Agha, par exemple, pour laquelle il construisit jardins et palais ${ }^{93}$, et qui est peut-être représentée dans la miniature de l'Aq-Saray, et bien sûr la mère de Shāh Rukh, Taghay Tärkän Agha, représentée lors de la naissance du prince ${ }^{94}$.

Trois destrente-quatre miniatures sont enfin consacrées à la chasse, loisir aristocratique par excellence, à laquelle s'adonnent princes et émirs, à l'instar de leurs héros Bahrām Gūr ou Kay Khusraw. Le divertissement a pour but de tester l'agilité et l'adresse des émirs ou des chefs d'armée, comme le montrent Amīr Shaykh Nūr al-Dīn chassant le lion en février

86. Zafarnāme, I, p. 368

87. Zafarnāme, p. 291. SIMS 1990-1991, fig. 4, p. 185, f. 160r, collection privée.

88. Zafarnāme, vol. 2, p. 20.

89. Zafarnāme, p. 244-250. SIMS 1990-1991, fig. 3, p. 185. Folio 139v, Settignano (Florence), Villa I Tatti. The Harvard University Center for Italian Renaissance Studies, Berenson Collection.

90. SIMS 1990-1991, fig. 11/12, p. 188. Folios 249v-250r, Washington D.C., Smithsonian Institution, The Freer Gallery of Art, 48.18 .

91. Notons que Claude HAASE (1981, p. 67) avait proposé de rapprocher deux miniatures des femmes de Tīmūr, celle représentant Balqīs et Salomon, n² 255, TSM, H2160, f. 38r et l'autre n 49, H 2160, f. 77v, Hazine 2153, f. 3v-4r, un cortège princier mettant en scène une fiancée, femme ou fille de Tīmūr.

92. CLAVIJO, La Route de Samarkand, p. 225, 233, 238.

93. Zafarnāme, livre 1, p. 298.

94. Ibid., fig. 4, p. 185 ; Zafarnāme, p. 409. SIMS 1990-1991, fig. 6, p. 186, f. 179v, Seattle, Seattle Art Museum, 49.133, Eugene Fuller Memorial Collection. 
$1399{ }^{95}$ devant Tīmūr, ou Tīmūr chassant près de Boukhara après la campagne de printemps de $13899^{96}$, ou dans la Jazīra à Ra's al-'Ayn en mars $1401{ }^{97}$.

Outre la famille, les miniatures dédiées à Tīmūr témoignent aussi dans une certaine mesure de la mutation profonde des modes de vie princiers à l'époque timouride. Seminomades, les Bārlās dédaignent visiblement la sédentarisation, et Tīmūr lui-même, sur trente-six années de pouvoir, en passa une quinzaine dans des campements militaires avant de séjourner à Samarkand. C'est d'ailleurs dans un vaste campement que se tinrent les festivités de mariage de ses petits-fils, dont les miniatures d'un folio issu d'une copie tardive conservée à Istanbul donnent une idée en montrant Tīmūr en majesté devant sa tente et le quartier des épouses tel que Clavijo le décrit ${ }^{98}$.

En fait, à Samarkand, Tīmūr fit édifier un grand nombre de jardins évoqués en détail par ibn 'Arabshāh ${ }^{99}$ et Clavijo ${ }^{100}$ qui, lui, connut les splendeurs du Bagh-i Dilgushā. Ces jardins, pourvus de parterres géométriques et d'essences de fruits et de fleurs très variées, constituaient de véritables oasis où l'on pouvait construire des pavillons abritant les familiers. Chacune de ses épouses ou concubines favorites possédait son propre jardin et Tīmūr, lors de ses séjours à Samarkand, s'y déplaçait en permanence. Le jardin clos, peuplé d'arbres fruitiers ou d'arbustes à fleurs, est lui-même devenu un lieu de prédilection des princes, un substitut des lieux de plein air où le prince accueille ses amis, reçoit ses épouses, se délasse, lit, etc. Le jardin timouride, étudié dans de nombreux travaux, donne une dimension particulière à la scène du premier plan, soulignant, tel un écrin, les personnages représentés ${ }^{101}$.

Quant aux palais, on en trouve l'évocation discrète dans une miniature présentant l'inauguration de l'Aq-Saray en 1379, dans une scène de cour. Le palais est un lieu clos, peu apprécié, que le souverain abandonne à ses artisans, au profit de la tente promue symbole $\mathrm{du}$ pouvoir à la fois politique, militaire et familial ${ }^{102}$. Les tentes, dont l'ambassadeur espagnol Clavijo nous fit une éloquente description, sont déployées dans ces mêmes jardins construits pour ses femmes, ses petites-filles, en dehors des enceintes urbaines. Il est ainsi révélateur de voir des tentes dessinées par les artistes timourides à l'intérieur du mythique palais de Khawarnaq dans la Khamsa de Niẓāmī ${ }^{103}$. Comme l'a noté Michele Bernardini, Tīmūr fit étrangement subir aux deux architectes du Bibi Khanūm, Muḥammad

95. Zafarnāme, p. 75 ; SIMS 1990-1991, fig. 16, p. 189 : f. 305v, localisation inconnue.

96. Zafarnāme, p. 58-59 ; SIMS 1990-1991, fig. 7, p. 186 : f. 196v, Houston, Texas, Soudavar Collection.

97. SIMS 1990-1991 fig. (19) 20, p. 190, f. (341v)-342v, The Montreal Museum of Fine Arts, 962. Ea38 (Dr. 1986.108), The F. Cleveland Morgan Bequest.

98. Zafarnāme, 1486, Turk ve Islam Ezerleri Muzesi, dans ROXBURGH 2005, fig. 172, p. 220.

99. Ibn 'Arabshāh, Tamerlane or Timur the Great Amir, p. 310.

100. CLAVIJO, La Route de Samarkand, p. 207-217.

101. GOLOMBEK 1995, p. 137-147.

102. GRONKE 1992, p. 18-22 ; O'KANE 1993, p. 249-269.

103. GRONKE 1992, p 19. 
Jild et Mahmūd Dāwūd, le même sort que naguère le roi du Yémen Nu'mān à Sinimmār, l'infortuné architecte grec du fabuleux palais de Khawarnaq : ils furent exécutés ${ }^{104}$ !

Une seule dimension est négligée dans la miniature: la religion. Pourtant, sous l'impulsion de Shāh Rukh promu chef de famille à la mort de Tīmūr, le texte du Zafarnāme avait en effet mis l'accent sur la piété musulmane de Tīmūr, en insistant sur son intérêt pour les hommes saints et pieux. Assez peu amateur de plaisirs, voire puritain, Shāh Rukh transféra sa capitale à Hérat. Il rompit en apparence avec les traditions politiques turcomongoles et voulut régner comme un monarque musulman, préférant le titre de "Sulțān » à celui de « Kürägän »; cette connotation strictement islamique se retrouve par ailleurs dans ses choix bibliophiliques ${ }^{105}$. On connaît son intérêt pour l'Histoire des Mongols de Rashīd al-Dīn dont il collecta des manuscrits à des fins historiographiques ; il en fit exécuter des copies enluminées à l'image de trois d'entre elles conservées à Istanbul à Topkapi, et reproduisant des scènes historiques (par exemple le ms. H. 1654) ${ }^{106}$; par ailleurs, ses commandes de manuscrits et de construction d'édifices religieux furent également suivies par tous ses fils ${ }^{107}$. Le but de Shāh Rukh était de réconcilier la dynastie avec les élites religieuses de l'Iran et d'apaiser les conflits attisés par les sectes sous une image de souverain charismatique et inspiré ${ }^{108}$; d'où peut-être la volonté du souverain de policer la maison royale, en commençant par son fils Muhāmmad Jukī (1402-1444), grand amateur de manuscrits mais débauché notoire.

\section{LES PRINCES TIMOURIDES, HÉROS D'ÉPOPÉE?}

Si l'image de Tīmūr devait servir prioritairement de miroir aux princes pour ses petitsfils, ces derniers surent également trouver d'autres modèles édifiants, correspondant sans doute davantage à leur sensibilité et à leur imaginaire : les héros de l'Iran ancien glorifiés par la littérature épique.

\section{1) La fascination familiale pour les héros du passé}

Si l'art du livre connut une période de faste sans précédent, c'est en raison de l'intérêt des princes timourides avides d'adhérer aux valeurs culturelles du monde persan dont ils adoptent sans réserve la langue, les valeurs aristocratiques, le mode de vie, tels que les véhiculent les œuvres épiques rédigées du $\mathrm{X}^{\mathrm{e}}$ au XII ${ }^{e}$ siècle par les poètes persans au service de dynastes turcs comme eux. En effet, lorsque Firdawsī composa le Shāhnāme, pour le turc Mahmūd de Ghazna, il ne se doutait pas de la fortune de l'œuvre, véritable miroir au prince pour les rois mongols et turcs après eux.

104. BERNARDINI 1992, p. 36-37. Sur le palais et l'architecture timouride, voir GOLOMBEK et WILBER 1988.

105. SUBTELNY 1983, p. 127-128.

106. INAL 1992, p. 103-115.

107. LENTZ et LOWRY 1989, p. 92-93.

108. FORBES MANZ 2007, p. 191. 
Tīmūr eut un double rôle dans cet essor culturel : il ramena en effet avec lui bon nombre d'œuvres d'arts, de manuscrits, mais aussi d'artistes et d'artisans de l'ouest en captivité en Asie centrale ; ces derniers œuvrèrent à Chiraz, Samarkand ou Hérat, au service de ses fils et petits-fils et, par leurs talents conjugués, permirent le développement de brillantes écoles de peinture. Par ailleurs, ses exploits, présentés historiquement comme une sorte de continuation de l'entreprise gengiskhanide, furent symboliquement reliés aux entreprises épiques, et Tìmūr fut promu au rang des héros qui combattirent avant lui les ennemis séculaires de l'Iran: Turcs (Bahrām Gūr, Rustam), Slaves, Chinois ou Indiens (Iskandar, Isfandiyār). Le ShāhanShāhnāme (ms. B.L. Or. 2780), exécuté à Chiraz vers 1397-98, et dont les illustrations mêlent à la fois thèmes historiques et thèmes mythiques, en est la preuve ${ }^{109}$.

Le Livre des rois fut l'ouvrage de prédilection des princes timourides, ardents commissionnaires de manuscrits illustrés. Les grands poètes persans sont également appréciés par les princes timourides, Nizāāmī de Ganja au XII ${ }^{\mathrm{e}}$ siècle en particulier qui, dans la Khamsa, raconte là encore les exploits du prince Bahrām Gūr, mais aussi les histoires d'amour éternelles, Khusraw et Shìrin, Layla et Majnūn, l'Iskandarnāme ou les exploits d'Alexandre le Grand, et enfin un poème spirituel, le Trésor des secrets. Quête terrestre et quête spirituelle se trouvent ainsi résumées, exposées dans des vers que les princes connaissent par cœur. La Roseraie des mystères, le Dialogue des oiseaux et divers autres poèmes sont aussi mis en peinture et calligraphiés pour eux ${ }^{110}$.

Comme l'a montré E. Sims, les ouvrages commissionnés par les princes timourides reflètent sinon un programme iconographique prédéfini, tout au moins les grandes tendances de leur conception du pouvoir, à l'instar de leurs exemplaires respectifs du Shāhnāme (Planche 2). Si tous sont hommes de guerre, l'activité militaire semble avoir été celle de prédilection d'Ibrāhīm Sulțān ${ }^{111}$, alors que Muhammad Jukī préférait les héros d'épopée et leur monde imaginaire. Pour ne prendre qu'un exemple précis, Bāysunghur Mirza (m.1433), l'un des fils de Shāh Rukh, affectionne visiblement les scènes de souveraineté, qui mettent l'accent sur la légitimité du pouvoir ${ }^{112}$.

\section{2) Bāysunghur Mirza, prince courtois (1397-1434)}

Nommé par son père gouverneur de Hérat, fonction qu'il assura jusqu'en 1434, Bāysunghur est un lettré notoire dont on possède aujourd'hui les moraqqa ou exercices de calligraphie ${ }^{113}$ (Istanbul, TSM, Hazine 2152, f.31B, Hérat, 1420-1430). Il fut le commanditaire d'un grand nombre d'ouvrages, puisque l'on possède encore une quarantaine de ses manuscrits enluminés, et il fut certainement le commanditaire réel de certains manuscrits de Shāh Rukh. Sur l'ensemble des manuscrits connus, dix-huit chefs-

109. FORBES MANZ 2007, p. 59. Voir note 2.

110. BARRY 2000 et 2004 ; SUBTELNY 1986, p. 56-79.

111. SOUCEK 1998, p. 32-36. Voir frontispice du Shāhnāme, Add. 176, Oxford, Bodleian Library, Hérat, 1430 env., f. $6 \mathrm{v}-7 \mathrm{r}$.

112. SIMS 1992, p. 44-68.

113. LENTZ et LOWRY 1989, cat. 39, p. 115. 
d'œuvre identifiés portent la kunya «al-bāysunghurī»; en outre, il correspondait avec son frère Ibrāhīm Sultān, gouverneur de Chirāz, autre mécène de l'art du livre. Il hérita d'une partie des artistes de son cousin Iskandar Sultān en 1414, dont le peintre 'Abd al-'Âlî Naqqāsh, le calligraphe Márūf Baghdadī et le relieur Khwajah Mahmūd. Le rapport sur le fonctionnement de son kitabkhāne rédigé par Ja'far al-Tabrīzī est désormais bien connu ${ }^{114}$. À sa mort, son fils hérita de son atelier qui fonctionna jusqu'en 1447.

Le prince fit orner un grand nombre de manuscrits, dont le Jāmi al-tawärikh de Paris que son père avait fait copier par Sayf al-Vahadī, un poète et enlumineur de sa cour à Hérat vers $1430{ }^{115}$, mais aussi un magnifique Shāhnāme, réalisé à Hérat en 1430 et conservé au Gulistān Palace à Téhéran, particulièrement représentatif puisque le prince y est présenté en frontispice chassant le lion et l'onagre ${ }^{116}$. Les thèmes des poèmes et épopées sont ainsi repris dans la mise en scène des portraits supposés de Bāysunghur, promu par ce biais aristocrate modèle de son temps. Livré à des activités de loisirs, on le voit ainsi chassant un loup dans une vaste composition réalisée à Hérat en $1425{ }^{117}$, mais aussi dans une vaste composition d'un double frontispice d'un Shāhnāme copié en $1430{ }^{118}$. Par ailleurs, il est représenté en majesté dans le frontispice d'un Kalīla et Dimna illustré en $1429^{119}$, et dans le folio d'une anthologie conservée à Florence, dans un jardin, assis au milieu de ses épouses ${ }^{120}$. Mīr Dawlathshāh Samarqandī, qui officiait alors à la cour de Shāh Rukh, le décrit comme " possédant beauté et perfection, chanceux, patron des arts et des lettres ${ }^{121}$ ».

Les analogies visuelles entre les thèmes choisis par les Timourides pour leur portrait et ceux des Shāhnāme sont patents. Du reste, si le choix du portrait intégré dans les frontispices n'est pas inhabituel dans l'art islamique, il n'en demeure pas moins que les princes timourides en systématisent la pratique qui prend alors valeur de symbole, le prince étant à la fois commanditaire du manuscrit et intégré comme héros potentiel ${ }^{122}$.

En outre, les Timourides, conscients de l'impact de l'image quant à la constitution d'une idéologie princière dominante, font aussi réaliser des fresques murales dans leurs palais, dévoilant leurs idées, leurs goûts et leur grandeur, à l'image des fresques ornant, d'après Ibn 'Arabshāh ${ }^{123}$, les palais de Tīmūr lui-même, et ce dans la tradition certes connue des cours sassanides, mais renouant de façon plus récente avec les décors des palais ghaznévides tel celui de Lashkari Bazar, des fresques seljoukides, mais sans aucun doute aussi de l'influence des Kartides d'Hérat (1245-1389) qui auraient maintenu cette

114. Arzadasht de Ja far Tabrizī, trad. W. M. Thackston, dans LENTZ et LOWRY 1989, p. 364-65.

115. Rashīd al-Dīn, Histoire des Mongols, Paris, BnF. sup. persan 1113, Hérat, 1430-1434 env.

116. SIMS 1992 p. 54, fig. 16.

117. LENTZ et LOWRY 1989, cat. 33, p. 113.

118. LENTZ et LOWRY 1989, Shāhnāme, Hérat, 1430, Téhéran Gulistan Palace, ms. 61, fig. 42, p. 132.

119. LENTZ et LOWRY 1989, Kalīla et Dimna, Hérat, 1429, Istanbul, TSL, R. 1022, p. 135-137.

120. Lentz et Lowry 1989, Anthologie, Hérat, 1426-1427, Florence, Villa I Tatti, Berenson Collection, fig. 40, p. 124.

121. Mīr Dawlathshāh Samarqandī, Tadhkirat, dans THACKSTON 1989, p. 22b.

122. SOUCEK 2000, p. 105.

123. LENTZ 1993, p. 263. 
tradition aulique dont les Timourides purent s'inspirer directement ${ }^{124}$. Ainsi, toutes les représentations, qu'elles soient enluminures ou fresques murales, ne valorisent pas seulement le prince dans sa dimension potentiellement héroïque, mais sont également utilisées pour exposer publiquement son programme et ses choix politiques.

\section{3) Les princes timourides et les astres}

Iskandar Sulțān, l'un des fils de 'Umar Shaykh (m. 1394), fut promu gouverneur du Fars et il s'installa à Ispahan en 1412 puis, marié à une fille d'Ahmad Jalayr, il bénéficia du travail de certains artistes œuvrant à Bagdad, parmi lesquels 'Abd al-'Ālì Naqqāsh dont Bāysunghur hérita en 1414 à Hérat.

Soucieux lui aussi de la mémoire familiale, il est visiblement le commanditaire d'une généalogie synoptique, dont l'auteur est inconnu, écrite en 1413 et conservée dans un compendium lui ayant appartenu ${ }^{125}$ (TSL, B411, f $\left.{ }^{\circ} 159 \mathrm{a}\right)$. L'auteur anonyme passe presque sans transition de Tīmūr à Iskandar Sulțān, signalant à peine l'intermède des quatre années de règne de Miranshāh. Il fait pour ainsi dire apparaître Iskandar Sultān comme le chef de famille alors que son oncle Shāh Rukh exerçait cette fonction. L'auteur précise qu'il établit ses frères dans toutes les régions conquises sur les Muzaffarides du Fars ou sur les ayants droit de Miranshāh afin qu'ils contrôlent toutes les provinces.

« (...) celle du Luristan, et les autres provinces jusqu'aux portes de Tabriz, et de la Mecque à

Médine, jusqu'à Kush et Makran, et depuis le Khurasan jusqu'aux portes d'airain de Darband, tout était sous le contrôle des gouverneurs à son service ${ }^{126}$. »

En reprenant l'homonymie Alexandre - Iskandar, l'auteur anonyme évoque la muraille d'airain jadis édifiée par Alexandre le Bicornu pour contenir les Gog et Magog, la suggérant comme limite tangible du pouvoir du jeune prince ${ }^{127}$. Pourtant, les Gog et Magog sont ici convertis en Moghols de l'Inde, nouveaux ennemis de l'État timouride. La curieuse évocation des peuples servant l'antéchrist trouve une explication chez Mīr Dawlathshāh Samarqandī qui précise, quant à lui, que le jeune prince, fier de sa splendeur et de son pouvoir, récitait des vers à sa propre gloire dont le sens est approximativement celui-ci :

Quelle importance les peuples de Gog et Magog ou les événements mondiaux peuvent-ils avoir pour moi qui suis fort et splendide tel le barrage d'Alexandre ${ }^{128}$ ?

Gouverneur de Shirāz et au faîte de sa gloire, Iskandar Sulțān fut le commanditaire de nombreux ouvrages littéraires et scientifiques, dont une anthologie conservée à Lisbonne, une anthologie scientifique à Londres et une autre à Istanbul, qui comportent toutes des

124. DIBA 2001, p. 7-11.

125. Anonymous, A Synoptic Account of the House of Timur, dans THACKSTON 1989, p. 237-246.

126. Anonymous, A Synoptic Account of the House of Timur, dans THACKSTON 1989, p. 241a.

127. BACQUÉ-GRAMMONT, DE POLIGNAC et BOHAS 2000, p. 109-127.

128. Mīr Dawlathshāh Samarqandī, Tadhkirat, dans THACKSTON 1989, p. 30. 
tables astronomiques et des traités d'astrologie ${ }^{129}$, mais aussi un magnifique horoscope enluminé qui trahit son désir de pouvoir.

L'horoscope d'Iskandar reproduit le ciel au moment de ses trente ans, donc au faite de sa puissance, et révèle que le jeune prince s'empare du pouvoir, ce qu'il va payer de sa vie. L'intérêt des princes timourides pour les astres semble aujourd'hui établi, comme le montre la présence de l'astrologue à la naissance de Ghāzān. Il établissait les thèmes des princes, était sollicité pour la construction de palais ou jardins, pour entreprendre des expéditions militaires. Toutefois, les détails que l'examen de la miniature révèle ressemblent fort à une sorte d'investiture céleste du pouvoir : les anges reconnaissant Iskandar Sultān comme dynaste, lui apportent, outre des présents, la bénédiction des cieux et celle des astres le couronnant ${ }^{130}$.

Ce rapport au monde n'est au fond pas différent du mythe de la royauté cosmique telle que le poète Nizāāī la présente dans la geste du roi Bahrām Gūr et des sept princesses : chaque jour, en visitant une princesse différente, il reçoit une initiation spirituelle dispensée par la princesse, « ange » de la planète initiatrice. L'anthologie de Lisbonne montre ainsi le prince Bahrām contemplant dans les cieux la voie de son destin ${ }^{131}$.

Iskandar Sulțān demeure l'une des personnalités princières les plus attachantes. Cultivé, mais ambitieux, voulant le titre de «Sultān » pour lui-même, il désobéit à son oncle Shāh Rukh, et fut soumis pour rébellion en 1413, puis aveuglé en juillet $14144^{132}$.

Ulugh Beg est, quant à lui, l'un des fils de Shāh Rukh et, contrairement à son cousin Iskandar Sulțān, il fut toujours fidèle et soumis à son père ${ }^{133}$. Mīr Dawlathshāh Samarqandī le décrit comme un prince accompli, doté de grandes qualités intellectuelles, dont une mémoire prodigieuse et un goût pour les sciences mathématiques et astronomiques. Il fut à la fois homme de guerre, homme de sciences et de culture car, lorsqu'il attaqua l'armée mongole en 1425, il n'oublia pas de rapporter un nombre impressionnant d'objets en jade, dont la pierre tombale de son grand-père ${ }^{134}$.

Ulugh Beg est célèbre aujourd'hui encore ${ }^{135}$ pour ses brillantes études d'astronomie dont les $Z \bar{i}$ j- $i$ Gurgānī, une révision des tables d'étoiles ptoléméennes, mais aussi pour la construction d'un observatoire à Samarkand dont il ne reste plus que l'arc méridien, et qu'il fit peut-être bâtir d'après le modèle de celui de Marāgha où Nāṣīir al-Dīn al-Tūsī avait travaillé. Parmi les personnalités les plus célèbres l'entourant, Jamshīd al-Dīn al-Kashī, autrefois au service d'Iskandar Sulțān, et le soufi Qazizada Rūmī.

On possède encore un globe céleste en laiton, conservé au British Museum, datant de 1430, et, d'après 'Abd al-Razzāq Samarqandī, sur les murs de l'observatoire auraient été peintes des représentations des cieux, les sept planètes, les étoiles fixes, etc. Le splendide

129. RICHARD 1996, p. 45-72 ; SOUCEK 1992, p. 116-131.

130. CAIOZZO 2005, p. 115-144.

131. GRAY 1979, p. 75.

132. Mīr Dawlathshāh Samarqandī, Tadhkirat al-Su'ara, dans THACKSTON 1989, p. 31a.

133. BARTOLD 1959.

134. L'époque est marquée par le goût prononcé pour les chinoiseries, voir entre autres Islamic Art, I, 1989 et CROWE 1992, p. 168-178.

135. KENNEDY, 2000, p. 97-110 et YULDASHBAEV 2000 p. 139-142. 
manuscrit des Étoiles Fixes de Paris ${ }^{136}$ (Paris, BnF. Arabe 5036) possède son autographe et son portrait supposé sous les traits de la constellation de Céphée (Planche 3) en raison de la forte analogie avec le portrait identifié conservé à la Freer Gallery of Art ${ }^{137}$.

Ulugh Beg demeure cependant un prince turco-mongol qui, dans la droite ligne de son grand-père, applique le yasa, épouse des princesses mongoles et garde le titre de kürägän. Il embellit Samarkand dont il fut le maître durant quarante ans, en organisant le Registān, sa place monumentale. Mais, pour finir, il fut traîtreusement assassiné à l'instigation de son propre fils, 'Abd al-Lāțîf, unanimement condamné par l'historiographie timouride ${ }^{138}$.

Sur le modèle d'Ulugh Beg et de ses fils, les princes timourides s'entre-déchirèrent, si bien que de 1447 à 1458, une nouvelle horde de Turcs, dont Jahanshāh Qaraqoyyunlu, entrèrent à Hérat et saccagèrent la ville. Le dernier timouride s'y réinstalla en 1470 et, jusqu'en 1506, régna en prince éclairé digne des premiers timourides, sous le nom de Sulțān Husayn Bayqara (1438-1506), arrière petit-fils de 'Umar Shaykh. Sous le règne de Sulțān Husayn, les mythes mis à l'honneur par les Timourides trouvèrent un nouveau souffle avec l'école dite de Behzad ${ }^{139}$. Outre de magnifiques exemplaires des épopées persanes, le Zafarnāme fit l'objet de nouvelles illustrations.

La gloire des Timourides ne s'éteignit pas de si tôt, puisque leur descendant en Inde, Babūr, au XVI ${ }^{\mathrm{e}}$ siècle, fier de ses origines et des mythes familiaux, rappela leurs exploits aux côtés des siens dans sa propre épopée, le Babūrnāmeh ${ }^{140}$. Toutefois, les Timourides avaient introduit bien des innovations culturelles dans l'art du livre. Outre leur implication personnelle comme calligraphes, collectionneurs et mécènes, ils surent donner au livre enluminé une dimension idéologique à travers le portrait que les Moghols, puis beaucoup plus tard les Qajars, reprirent dans leur sillage ${ }^{141}$. Cette alliance entre l'art et la politique invite aujourd'hui les historiens de l'art, et plus encore ceux de l'image, à une relecture en règle des corpus enluminés d'époque turco-mongole et leurs corollaires mamelouks dans une perspective d'ensemble où la miniature ne serait plus seulement un art princier mais un art guidé par les princes, ceux du passé préislamique, leurs modèles et ceux du présent, leurs héritiers. Au-delà, plus encore que l'omniprésence de la culture persane classique, l'engouement des princes turco-mongols pour les mythes héroïques persans invite à un questionnement sur la pérennité de ces mythes, voire leur adaptation et leur réemploi dans l'art du livre, dans une perspective élargie à l'ensemble des cours orientales sur la longue durée du XIII ${ }^{\mathrm{e}}$ au début du XVI ${ }^{\mathrm{e}}$ siècle.

\footnotetext{
136. CAIOZZO 2003, p. 66.

137. Ce portrait est très proche de celui d'Ulugh Beg sur un folio d'un manuscrit perdu, Washington, Freer Gallery of Art, ms reg. n 4626, pl. dans LENTZ et LOWRY 1989, p. 90, fig. 33. À ce sujet, voir ASHRAFI 1988, p. 24-30.

138. Mīr Dawlathshāh Samarqandī, Tadhkirat, dans THACKSTON 1989, p. 29-30.

139. SIMS 1996, p. 299-311.

140. Le Livre de Babur, p. 339.

141. SOUCEK 2000.
} 


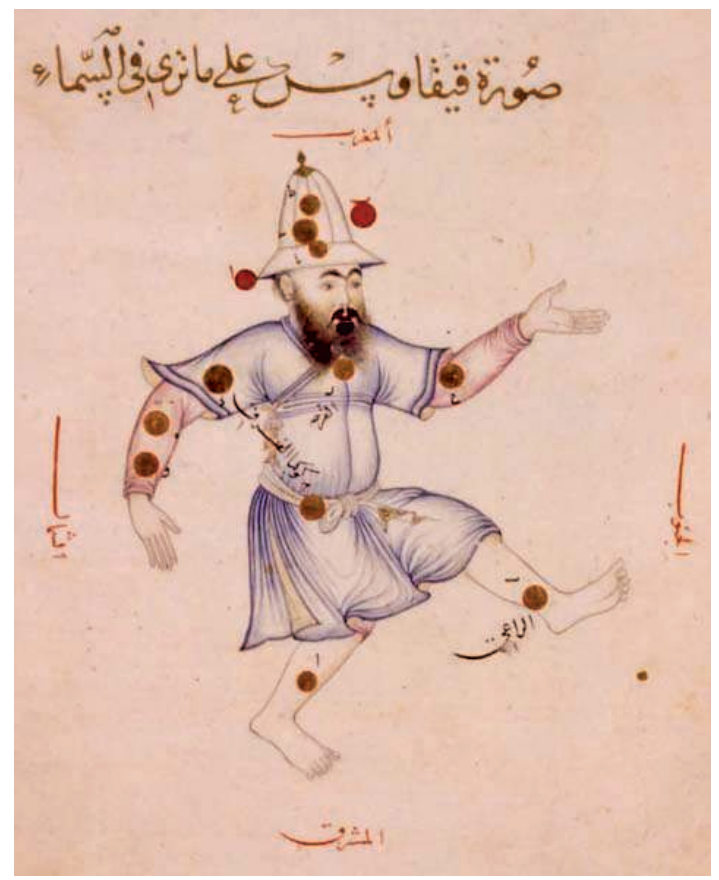

Planche 1 : la constellation Céphée dans le manuscrit commandité par le prince astronome Ulugh Beg b. Shāh Rukh, al-Ṣūfī, Livre des Étoiles Fixes, Paris, B.n.F., ms. arabe 5036, Hérat, 1440 env., f. 38 et 38v.

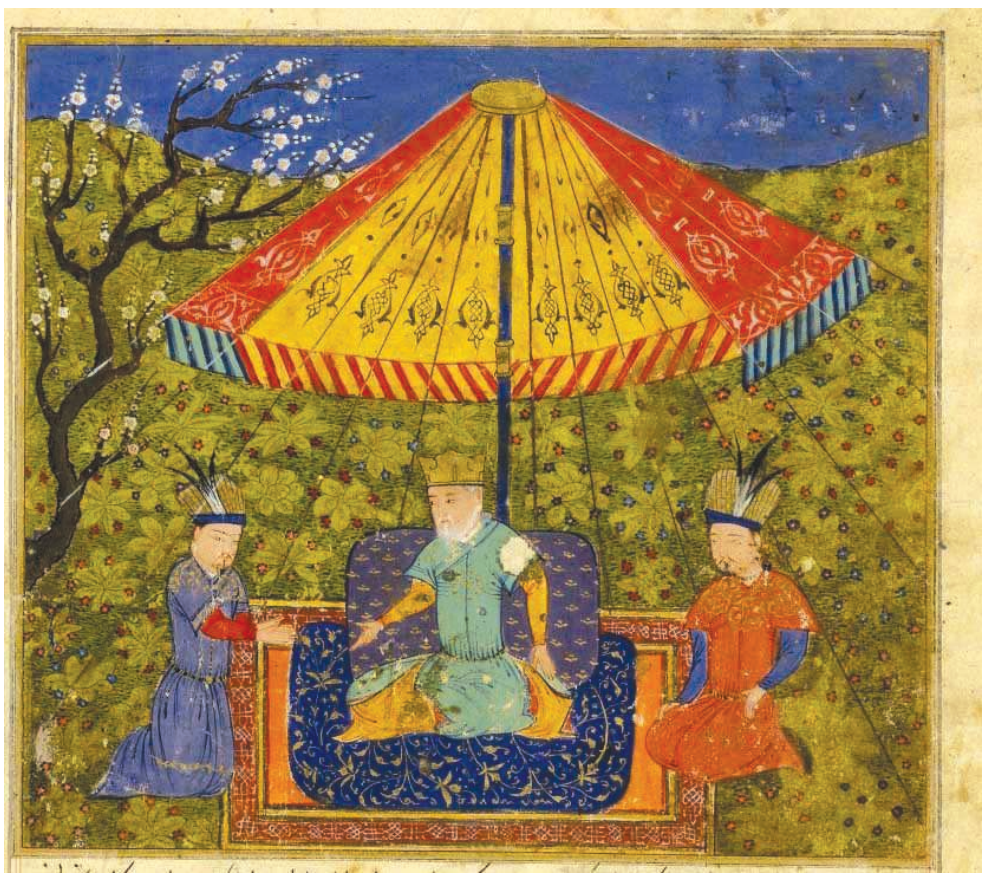

Planche 2 : Gengis Khān le modèle politico-militaire, Rashīd al-Dīn, Histoire des Mongols, Paris, B.n.F., ms. sup. persan 1113, Hérat, 1440 env., f. 116v. 


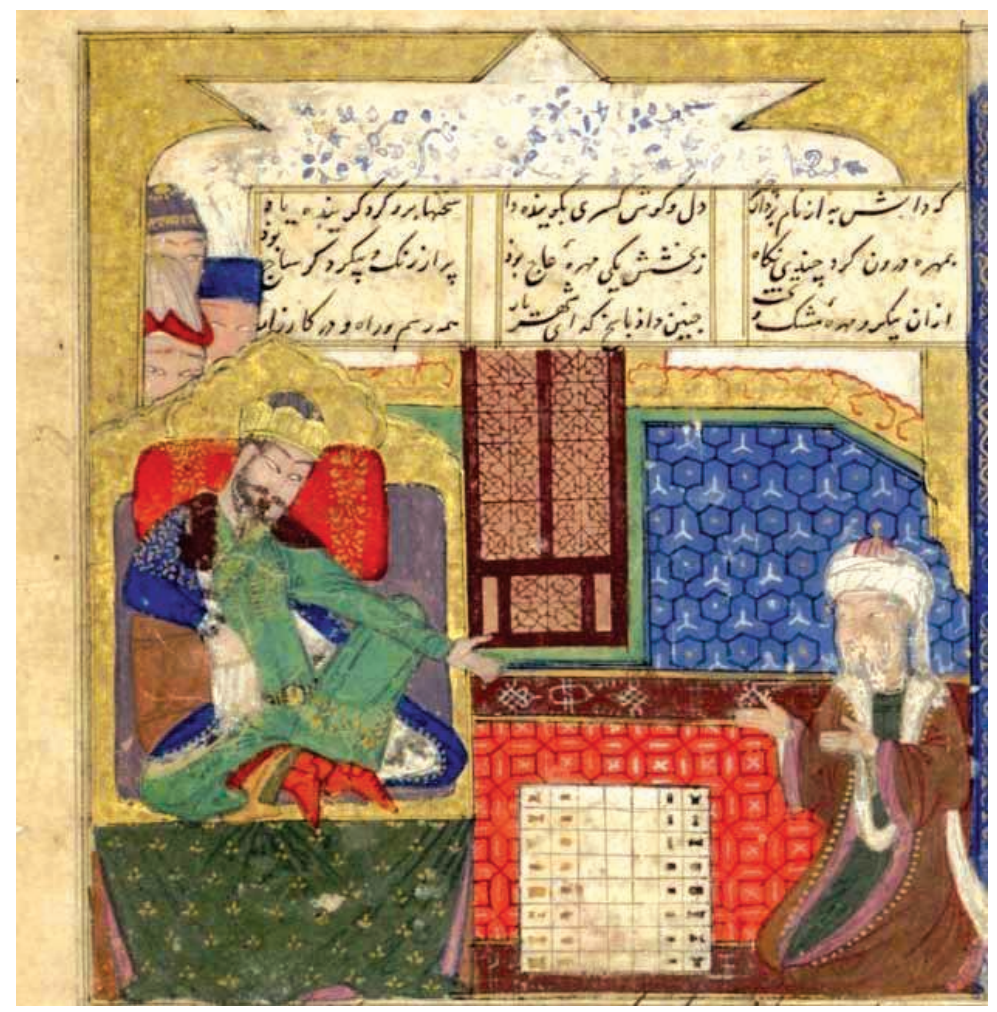

Planche 3: Khusraw Anūshirwān, le roi sage et instruit reçoit d'Inde le jeu d'échecs, Firdawsī, Shāhnāme, Paris, B.n.F., ms. sup. persan 493, Shirāz, f. 456.

\section{RÉFÉRENCES}

Sources

Clavijo, Ruy Gonzáles de, La Route de Samarkand au temps de Tamerlan, Relation du voyage de l'ambassade de Castille à la cour de Timour Beg, Ruy Gonzáles de Clavijo, 1403-1406, éd. et trad. L. KEHREN, Paris, Imprimerie nationale, 1990.

Ghiyāšsoddīn 'Alī di Yazd, Le gesta di Tamerlano, éd. et trad. M. BERNARDINI, Milan, Arnoldo Montadori Editore, 2009.

Ghiyāth al-Dīn 'Alī Yazdī, Sa'ādat-nāme yā Rūznāme-ye Ġazavāt-e Hendūstān, éd. Īraj AFŠĀR, Téhéran, $1379 / 2001$.

Histoire secrète des Mongols, Chronique mongole du XIII ${ }^{e}$ siècle, éd. et trad. M.-D. EVEN, R. POP, Paris, Gallimard, 1994.

Ibn 'Arabshāh, Tamerlane or Timur the Great Amir, éd. J. H. SANDERS, Lahore, Progressive Books, 1976.

Le Livre de Babur. Mémoires du premier Grand Mogol des Indes, 1494-1529, trad.J.-L. BACQUÉ-GRAMMONT, Paris, PUF, 1980.

Niẓām al-Dīn ‘Alī Shāmī, Zafar-nāme, éd. F. TAUER, Prague, Orientální ústav-Oriental institute, 1937-1956, 2 vol. 
Rashīd al-Dīn, Histoire des Mongols, Paris, BnF. sup. persan 1113, Hérat, 1430-1434 env.

Sharaf al-Dīn 'Alī Yazdī, Zafar-nāme, éd. M. ‘ABBĀSĪ, vol. I, Téhéran, 1957 ; vol. II, Tachkent, 1972.

Thaскston W. M. (éd. et trad.), 1989, A Century of Princes: Sources on Timurid History and Art, Cambridge (Mass.), The Aga Khan Program for Islamic Architecture.

Études

AIGLE D. (dir.), 2000a : Figures mythiques des mondes musulmans, dossier thématique dans Revue des mondes musulmans et de la Méditerranée 89-90. [En ligne] http://remmm.revues.org/1333

AIGLE D., 2000b : «Introduction : Le mythe créateur d'histoire », Figures mythiques des mondes musulmans, Revue des mondes musulmans et de la Méditerranée, 89-90, p. 7-38. [En ligne] http://remmm.revues. org $/ 271$

AIGLE D., 2000c: «Les transformations d'un mythe d'origine: L'exemple de Gengis Khân et de Tamerlan", Revue des mondes musulmans et de la Méditerranée, 89-90, p.151-168. [En ligne] http://remmm.revues.org/277

AIGLE D., (à paraître) : The Mongol Empire between myth and realities : historic anthropological studies, Leiden, Brill.

Amitai-Preiss R., 1996 : «Ghāzān, Islam and Mongol Traditions : A View from the Mamluk Sultanate », Bulletin of the School of Oriental and African Studies, 59/1, p. 1-10. DOI:10.1017/S0041977X00028524

ARBERRY J. et al., 1959: The Chester Beatty Library: A Catalogue of the Persian Manuscripts and Miniatures, vol. 1, Dublin, Hodges Figgis and Co.

ARNold Sir TH. W, 1930 : Bihzād and his paintings in the Zafar-nämah ms., Londres, Bernard Quaritch.

Ashrafi M. M., 1988 : «Where was the Portrait of Ulugh Beg Painted », Iranian Studies 21/1-2, p. 24-30.

Aubin J., 1963 : «Comment Tamerlan prenait les villes », Studia islamica 19, p. 83-122.

Bacqué-Grammont J.-L., Polignac F. de, Bohas G., 2000 : « Monstres et murailles, Alexandre le bicornu, mythe et bon sens. Quelques notes ", Revue des Mondes Musulmans et de la Méditerranée 89-90, p. 109-127. [En ligne] http://remmm.revues.org/275

BARRY M., 2000 : Painter, Poet, King and Saint: Bihzād, Jāmī, Nizāmī, and the Sanctification of Figurative Painting in Late Timurid Hérat, Washington, Freer Gallery (Occasional Papers).

BARRY M., 2004 : L'Art figuratif en islam médiéval et l'énigme de Behzād de Hérat (1465-1535), Paris, Flammarion.

BARTOLD V. V., 1959 : Four Studies on the History on Central Asia, vol. II, Ulugh Beg, transl. V. and T. MINORSKY, Leiden, Brill.

Blair S. S. 1996 : « Timurid Signs of Sovereignety », Oriente moderno (n. s., année 15) 76/2, p. 551-576.

BERNARDINI M., 1992 : « Aspects littéraires et idéologiques des relations entre aristocratie et architecture à l'époquetimouride »,dansGOLOMBEK L.,SUBTELNYM.E.(eds.),TimuridArtandCulture, IranandCentral Asia in the Fifteenth Century, Leiden, Brill, p. 36-43.

BERNARDINI M., 2008 : Mémoire et propagande à l'époque timouride, Paris, Société pour l'avancement des études iraniennes.

CAIozzo A., 2003 : Images du ciel d'Orient au Moyen Âge, Paris, PUPS.

CAIozzo A., 2005 : «The Horoscope of Iskandar Sultān as a Cosmological Vision in the Islamic World », dans Oestmann G., Rutkin H. D., Stuckrad K. von (eds.), Horoscopes and Public Spheres, Actes du colloque d'Amsterdam, 2004, Berlin - New York, Walter de Gruyter (Series: Religions and Society), p. 115-144.

CRowe Y., 1992 : « Some Timurid Designs and their Far Eastern Connections », dans GolombeK L., SubTelny M., (eds.), Timurid Art and Culture. Iran and Central Asia in the Fifteenth Century, Leiden, Brill p. 168-78. 
DiвA L. S., 2001: «Invested with Life: Wall Painting and imagery before the Qajars », Iranian Studies 34/3-4, p. 5-16.

Dobrovits M., 1994: «The Turco-Mongolian Tradition of Common Origin and the Historiography in Fifteenth Century Central Asia », Acta Orientalia 47, p. 269-277.

Fischel Walter J., 1952 : Ibn Khaldûn and Tamerlane, Their Historic Meeting in Damascus, 1401 A.D. (803). A Study based on Arabic Manuscripts of Ibn Khaldûn's " Autobiography ", Los Angeles, University of California Press.

Forbes MANz B., 1988 : « Tamerlan and the Symbolism of Sovereignety », Iranian Studies 21/1-2, p. 107-118.

Forbes Manz B., 1989 : The Rise and Rule of Tamerlane, New York, Cambridge University Press.

Forbes MAnz B., 2000 : «Mongol History rewritten and relived», Revue des mondes musulmans et de la Méditerranée 89-90, p. 129-149. [En ligne] http://remmm.revues.org/276

Forbes MANz B., 2002 : « Tamerlane's Career and Its Uses », Journal of World History 13,/1, p. 1-25.

Forbes MAnz B., 2007 : Power, Politics and Religion in Timurid Iran, Cambridge, Cambridge University Press.

Golomвек L., 1995 : « The Gardens of Timur: New Perspectives », Muqarnas 12, p. 137-147.

Golombeк L., Wilber D., 1988 : The Timurdi architecture of Iran and Turan, Princeton, Princeton University Press, vol. 1 et 2.

GRAY B., 1979 : La Peinture persane, Genève, Skira.

GronkeM.,1992 : «ThePersian CourtbetweenPalace and Tent:from Timur to Abbas Ist », dans GOLOMBEK L., SubTelny M. E. (eds.), Timurid Art and Culture, Iran and Central Asia in the Fifteenth Century, Leiden, Brill, p. 18-22.

Grousset R., 1965 : L’Empire des steppes, Paris, Payot.

GRUPPER S. M., 1995 : « A Barulas family narrative in the yuan-shih: some neglected prosopographical and institutional sources on timurid origins », Archivum Eurasiae Medii Aevi 8, p. 11-97.

HAASE C. 1981, « On the Attribution of Some Paintings in H. 253 to the Time of Timur », dans Islamic Art, 1981, New York, The Islamic Art Foundation, p. 50-65.

INAL G., 1992 : « Miniatures in Historical Manuscripts from the Time of Shah Rukh in the Topkapi Palace Museum », dans GOLOMBEK L.,SUBTELNYM.E.(eds.),TimuridArtandCulture, Iranand CentralAsiainthe Fifteenth Century, Leiden, Brill, p. 103-115.

Islamic Art, 1981-2001, New York, The Islamic Art Foundation.

Kennedy E. S., 2000 : « The Heritage of Ulugh Beg », dans IhsAnoĞLu E., Günergun F. (eds.), Science in Islamic Civilisation, Istanbul, Research Centre for Islamic History, Art and Culture (IRCICA), p. 97-110.

Lentz T. W., 1993 : « Dynastic Imagery in Early Timurid Painting », Muqarnas 10, p. 253-265.

LENTZ T. W., Lowry G. D., 1989 : Timur and the Princely Vision. Persian art and culture in the fifteenth century, Los Angeles-Washington D.C., Los Angeles County Museum of Art-Smithsonian Institute.

Mazzaou M. M., Moreen V. B. (eds.), 1990 : Intellectual Studies on Islam : Essays Written in Honor of Martin B. Dickson, Salt Lake City, University of Utah.

Melville C., 2006a : «Ibn Ḥusām's Ḩāvarān-nāma and the Šāh-nāma of Firdawsī », dans BeRnardini M., Haneda M., Szuppe M. (eds.), Liber Amicorum: Études sur l'Iran médiéval et moderne offertes à Jean Calmard = Eurasian Studies 5/1-2, p. 219-234.

Melville C., 2006b : « Keshig in Iran, The Survival of the Royal Mongol Household », dans Komaroff L. (ed.), Beyond the Legacy of Gengis Khan, Leyde, Brill, p. 150-155.

NecipoĞLu G., 2000 : «The Serial Portraits in Ottoman Sultans in Comparative Perspective », dans RABY J. (éd.), The Sultan's Portrait, Picturing the House of Osman, Istanbul, Isbank, 2000, p. 22-61.

O’Kane B., 1993 : « From Tents to Pavillions: Royal Mobility and Persian Palace Design », dans NecipoĞLu G. Pre-Modern Islamic Palaces $=$ Ars Orientalis 23, p. 249-269. 
Paul J., 1990 : «Scheiche und Herrscher im Khanat Cagatay », Der Islam 67/2, p. 278-321.

Polyakova E. A., 1988 : « Timur as Described by the 15th Century Court Historiographers », Iranians Studies 21/1-2, p. 31-44.

RichARD F., 1996 : «Un témoignage inexploité concernant le mécénat d'Eskandar Soltan à Esfahan », Oriente Moderno, n. s., année 15, 76/2, p. 45-72.

RICHARD F., 1997a : « Un des peintres du manuscrit supplément persan 1113 de l'Histoire des Mongols de Rashīd al-Dīn identifié », dans AIGLE D. (éd.), L’Iran face à la domination mongole, Téhéran, IFRI, p. 307-320.

RichaRD F., 1997b: Splendeurs persanes, Manuscrits du XII au XVII siècle (Catalogue d'exposition de la Bibliothèque nationale de France, Galerie Mazarine, 27 novembre 1997-1 ${ }^{\mathrm{er}}$ mars 1998), Paris, Bibliothèque nationale de France.

RoxвURGн D. J. (éd.), 2005 : The Turks, A Journey of Thousand Years, 600-1600, Londres, Royal Academy Of Arts.

SHIRo A., 1992: Timuridische Emire nach dem Mưizz al-ansāb, Berlin, K. Schwartz (Islamkundliche Untersuchungen 153).

Sims E., 1973: The Garett Manuscript of the Zafar-Nāme: A Study in Fifteenth-Century Patronage, P.H.D. Dissertation, New York, Institute of Fine Arts.

Sims E., 1990-1991 : «Ibrahim-Sultan's Illustrated Zafarnama of 839/1436», dans Islamic Art, vol. 4, New York, The Islamic Art Foundation, p. 175-218.

Sims E., 1992: «The Illustrated Manuscripts of Firdausî’s Shahnāma commissionned by Princes of the House of Tìmūr », Ars Orientalis 20, p. 44-68.

SIMs E., 1996 : «Sultan Husayn Bayqara's Zafarnamah and its Miniatures », dans The Memorial Volume of the $\mathrm{VI}^{\text {th }}$ International Congress of Iranian Art and Archeology, Oxford, September 11-16, 1972, Téhéran, p. 299-311.

Soucek P. P., 1992 : «The Manuscripts of Iskandar Sultan. Structure and Content », dans GoLombek L., SubTelny M., (eds.), Timurid Art and Culture, Iran and Central Asia in the Fifteenth Century, Leiden, Brill, p. 116-131.

SOucEK P. P., 1998 : «Ibrahim Sultan's Military Career », dans EsLami K. (ed.), Iran and Iranian Studies, Essays in Honor of Iraj Ashar, Princeton (N. J.), Zagros Press, p. 32-36.

Soucek P. P., 2000 : «The Theory and Practise of Portraiture in the Persian Tradition », Muqarnas 17, p. 97-108.

SoudAvar A., 2003 : The Aura of Kings, Legitimacy and Divine Sanction in Iranian Kingship, Costa Mesa, Mazda Publisher.

Subtelny M. E., 1983 : «Art and Politics in Early $16^{\text {th }}$ Century Central Asia », Central Asiatic Journal 27/1-2, p. 121-148.

Subtelny M. E., 1986 : « A Taste for the Intricate: the Persian Poetry of the Late Timurid Period », Zeitschrif der deutschen Morgenländischen Gesellschaft 136, p. 56-79. [En ligne] http://goo.gl/ikvTB

SuBtelny M. E., 2007 : Timurids in Transition. Turko-Persian Politics and Acculturation in Medieval Iran, LeidenBoston, Brill.

TrTLEY N., 1977 : Miniatures from Persian Manuscripts, Londres, British Library.

Woods J. E., 1987 : « The Rise of Timurid Historiography », Journal of Near Eastern Studies 46/2, p. 81-108.

Woods J. E., 1990 : « Timur's Genealogy », dans Mazzaou M. M. and MoreEN V. B., Intellectual Studies on Islam : Essays Written in Honor of Martin B. Dickson, Salt Lake City, University of Utah, p. 85-124.

Yuldashbaev T. S., 2000 : « Mirza Ulugh Beg and Modern Astronomy in Uzbekistan », dans IhsanoĞLu E., GÜNERGUN F. (eds.), Science in Islamic Civilization, Istanbul, IRCICA, p. 139-142. 
